\title{
The CIP method embedded in finite element discretizations of incompressible fluid flows
}

\author{
Bahareh Banijamali and Klaus-Jürgen Bathe ${ }^{*, \dagger}$ \\ Massachusetts Institute of Technology, 77 Massachusetts Avenue, Cambridge, MA 02139, U.S.A.
}

\begin{abstract}
SUMMARY
Quite effective low-order finite element and finite volume methods for incompressible fluid flows have been established and are widely used. However, higher-order finite element methods that are stable, have high accuracy and are computationally efficient are still sought. Such discretization schemes could be particularly useful to establish error estimates in numerical solutions of fluid flows. The objective of this paper is to report on a study in which the cubic interpolated polynomial (CIP) method is embedded into 4-node and 9-node finite element discretizations of 2D flows in order to stabilize the convective terms. To illustrate the capabilities of the formulations, the results obtained in the solution of the driven flow square cavity problem are given. Copyright (C) 2006 John Wiley \& Sons, Ltd.
\end{abstract}

Received 27 April 2006; Revised 23 October 2006; Accepted 23 October 2006

KEY WORDS: incompressible flows; finite element discretization; CIP technique; FCBI approach

\section{INTRODUCTION}

While much research has been done on the numerical solution of the Navier-Stokes equations, and solutions of very complex problems are obtained almost routinely in industry, there is still very important endeavour to develop more effective solution schemes, see e.g. [1-4] and the references therein. At present, the computational effort to solve a complex 3D problem can still be very large.

There are a number of challenges to reduce the numerical solution times of $3 \mathrm{D}$ fluid flow problems. The solution times of large given finite element models can be reduced, for example, by the development of improved parallel processing techniques, faster sparse and iterative solvers, and more effective domain decomposition methods. While these developments are clearly most valuable, it is, however, still a particular challenge to develop improved discretization schemes that are used with error measures so that the finite element meshes can be coarser and optimally

\footnotetext{
*Correspondence to: Klaus-Jürgen Bathe, Massachusetts Institute of Technology, 77 Massachusetts Avenue, Cambridge, MA 02139, U.S.A.

†E-mail: kjb@mit.edu
} 
structured. The premise is then that the number of unknown nodal variables in the discretizations can be kept small while the solution error is controlled. Of course, improvements in discretization schemes will also benefit all the other endeavours to render complex fluid flow analyses more efficient and hence more practical.

The use of coarse fluid flow meshes is in particular of much interest in fluid flow structure interaction problems. Here, the interest is frequently only on predicting a certain quantity-the goal quantity - in the structure accurately, like the stresses and the bending moment at the bottom of a blade of a power-generating windmill. The fluid flow does not need to be predicted very accurately in the complete fluid region as long as the forces on the structure are calculated with sufficient accuracy. For example, there seems to be no need to solve for very small eddies far away from the structure in order to predict the structural stresses accurately. However, in case a coarse mesh is used for the fluid flow, a measure of the numerical error made in the quantity of interest is very valuable $[5,6]$. Then, some control on the solution accuracy of the goal quantity is available. Clearly, the benefit of using a coarse mesh for the fluid flow prediction can be particularly large when a structural optimization is to be performed. Then an established and almost-proven adequate fluid mesh can be used throughout the structural optimization.

With the above aim in mind, we have pursued the development of fluid flow elements that are stable and always give reasonable solutions even when coarse meshes are used to model the fluid flow at high Reynolds numbers, as enumerated in References [7-11]. Of course, our aim is also that these elements should show optimal accuracy. The discretizations we developed are referred to as FCBI elements, because the convection terms are stabilized by use of flow-conditionbased interpolations. In practice the low-order elements are employed for steady-state and transient analyses, and they are effectively used together with an algebraic multi-grid solver [12].

However, as mentioned above, error measures to assess the solution accuracy can be important and in fluid flow structure interaction problems, when the aim is to only calculate a specific structural response accurately, goal-oriented error measures might be effective. In the error measure proposed by Grätsch and Bathe [6], 'influence functions' are used and they are best calculated using the same element subdivisions, first, with the low-order element and, then, with an effective higher-order element. Hence, for example, in 2D flow problems, a 4-node element might be used first and then a 9-node element. This 9-node element would ideally have excellent stability properties (to be used for very high element $R e$ numbers) and optimal accuracy (the same accuracy as provided by the Galerkin 9-node element in low Re number flows). We endeavoured to develop such 9-node element but the accuracy is not yet as high as we wish to obtain [11].

The objective in this paper is to present some further research results obtained to reach optimal 4-node and 9-node elements by drawing on the development of the cubic interpolated polynomial (CIP) method [13-16]. In this technique the advection part is interpolated using a cubic interpolation, the diffusion part is approximated using finite difference expressions and the combined equations are solved in a forward-stepping staggered manner. In the finite element formulations discussed in this paper, we also approximate the advection part by a cubic interpolation. Then for the 9-node element, to approximate the diffusion part, we use a mixed interpolation of velocities and pressure to satisfy the inf-sup condition for incompressible-flow analysis, and we use control volumes to locally satisfy mass and momentum conservation as in the FCBI approach. For the 4-node finite element, we solve for the diffusion part using the standard Galerkin method and a standard procedure for pressure correction.

In the following sections we first present the formulations of the new 4-node and 9-node elements, and then give the results obtained in the solution of the square cavity driven flow problem. 
The elements discussed show good stability and accuracy characteristics, but the computational efficiency is not sufficiently high. Further research to increase the efficiency is necessary as outlined in the conclusions of the paper and then the development should be applied in 3D analyses as well.

\section{FORMULATION OF THE 4-NODE ELEMENT}

In this section we present the formulation of the 4-node element for 2D incompressible fluid flows. The formulation could directly be extended for 3D flow solutions, but the purpose of this research was to first study the elements and identify their efficiency in 2D flows.

\subsection{The governing equations}

The non-dimensional momentum and continuity equations are written as

$$
\begin{aligned}
\frac{\partial \mathbf{v}}{\partial t}+(\mathbf{v} \cdot \nabla) \mathbf{v} & =-\nabla p+\frac{1}{R e} \nabla^{2} \mathbf{v} \\
\nabla \cdot \mathbf{v} & =0
\end{aligned}
$$

where $\mathbf{v}, p$ and $R e$ are the velocity vector, pressure and the Reynolds number, respectively. Here, we do not consider externally applied forces and temperature conditions.

In the CIP method, we also need the spatial derivatives of the momentum equations

$$
\begin{aligned}
& \frac{\partial \mathbf{v}_{x}}{\partial t}+(\mathbf{v} \cdot \nabla) \mathbf{v}_{x}=-\left(\mathbf{v}_{x} \cdot \nabla\right) \mathbf{v}-\nabla p_{x}+\frac{1}{R e} \nabla^{2} \mathbf{v}_{x} \\
& \frac{\partial \mathbf{v}_{y}}{\partial t}+(\mathbf{v} \cdot \nabla) \mathbf{v}_{y}=-\left(\mathbf{v}_{y} \cdot \nabla\right) \mathbf{v}-\nabla p_{y}+\frac{1}{R e} \nabla^{2} \mathbf{v}_{y}
\end{aligned}
$$

where the subscripts $x$ and $y$ signify the derivatives in the $x$ and $y$ directions, respectively.

We split the governing equations into an advection phase and a non-advection phase. The advection phase is solved by the CIP method and the non-advection phase is solved by a finite element scheme.

To use the CIP method we consider transient conditions in Equations (1) and (2). Then to reach a steady state, we need to continue the calculation until the solution is at steady state, that is, the velocities, pressure and the spatial derivatives of the velocities no longer change.

\subsection{The advection phase}

The governing equations of the advection phase in a typical time step are

$$
\begin{gathered}
\frac{\hat{\mathbf{v}}-\mathbf{v}^{n}}{\Delta t}+\left(\mathbf{v}^{n} \cdot \nabla\right) \mathbf{v}^{n}=0 \\
\frac{\hat{\mathbf{v}}_{x}-\mathbf{v}_{x}^{n}}{\Delta t}+\left(\mathbf{v}^{n} \cdot \nabla\right) \mathbf{v}_{x}^{n}=0 \\
\frac{\hat{\mathbf{v}}_{y}-\mathbf{v}_{y}^{n}}{\Delta t}+\left(\mathbf{v}^{n} \cdot \nabla\right) \mathbf{v}_{y}^{n}=0
\end{gathered}
$$




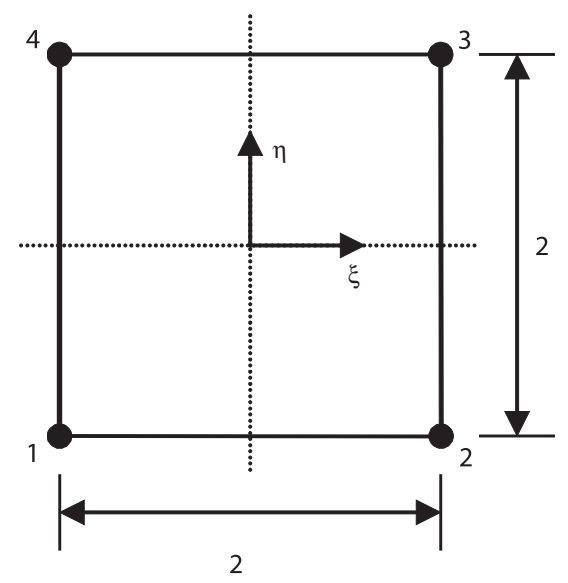

Figure 1. The 4-node element.

where $n$ denotes the beginning of the step, and $\hat{\mathbf{v}}, \hat{\mathbf{v}}_{x}$ and $\hat{\mathbf{v}}_{y}$ denote the velocity and velocity derivatives at the end of the advection phase step.

Consider the 1D advection equation for a potential $\phi$

$$
\frac{\partial \phi}{\partial t}+v \cdot \nabla \phi=0
$$

The solution at point $x_{i}$ and time ' $t$ ' can be approximated as

$$
\phi\left(x_{i}, t\right) \sim \phi\left(x_{i}-\int v \mathrm{~d} t, t-\Delta t\right)
$$

where $v$ is the particle velocity.

In general $2 \mathrm{D}$ analysis, $\int \mathbf{v} \mathrm{d} t$ is an 'advection displacement' and should be calculated along the particle path. The exact value of this displacement is difficult to obtain and it is approximated explicitly as an advection distance given by

$$
\Delta x=\left(\mathbf{v}^{n} \cdot \mathbf{e}_{x}\right) \Delta t, \quad \Delta y=\left(\mathbf{v}^{n} \cdot \mathbf{e}_{y}\right) \Delta t
$$

where $\mathbf{e}_{x}$ and $\mathbf{e}_{y}$ are unit base vectors in the $x$ and $y$ Cartesian co-ordinate directions. In order for this approximation to be close to the exact value, $\Delta t$ must of course be sufficiently small [17].

Using the CIP method, the potential $\phi$ is interpolated using third-order shape functions [18-20]

$$
\phi(\xi, \eta)=\sum_{a} h_{a}(\xi, \eta)\left[G_{a}(\xi, \eta)+E_{a}(\xi, \eta) \phi_{a}\right]
$$

where $h_{a}$ is the conventional bilinear shape function, and $G_{a}$ and $E_{a}$ are new shape functions that are second-order and linear, respectively. For the 4-node Galerkin-based element in Figure 1 these 
shape functions are, using the isoparametric co-ordinates $(\xi, \eta),[17,20]$

$$
\begin{gathered}
h_{1}=\frac{1}{4}(1-\xi)(1-\eta) \\
h_{2}=\frac{1}{4}(1+\xi)(1-\eta) \\
h_{3}=\frac{1}{4}(1+\xi)(1+\eta) \\
h_{4}=\frac{1}{4}(1-\xi)(1+\eta) \\
E_{1}=\frac{1}{2}(-\xi-\eta) \\
E_{2}=\frac{1}{2}(\xi-\eta) \\
E_{3}=\frac{1}{2}(\xi+\eta) \\
E_{4}=\frac{1}{2}(-\xi+\eta) \\
G_{1}=\frac{1}{2}\left(1-\xi^{2}\right)\left[\partial_{\xi} \phi_{1}+\phi_{1}\right]+\frac{1}{2}\left(1-\eta^{2}\right)\left[\partial_{\eta} \phi_{1}+\phi_{1}\right] \\
G_{2}=\frac{1}{2}\left(-1+\xi^{2}\right)\left[\partial_{\xi} \phi_{2}-\phi_{2}\right]+\frac{1}{2}\left(1-\eta^{2}\right)\left[\partial_{\eta} \phi_{2}+\phi_{2}\right] \\
G_{3}=\frac{1}{2}\left(-1+\xi^{2}\right)\left[\partial_{\xi} \phi_{3}-\phi_{3}\right]+\frac{1}{2}\left(-1+\eta^{2}\right)\left[\partial_{\eta} \phi_{3}-\phi_{3}\right] \\
G_{4}=\frac{1}{2}\left(1-\xi^{2}\right)\left[\partial_{\xi} \phi_{4}+\phi_{4}\right]+\frac{1}{2}\left(-1+\eta^{2}\right)\left[\partial_{\eta} \phi_{4}-\phi_{4}\right]
\end{gathered}
$$

where $\phi_{i}$ is the potential at node $i$.

We use this updating 'shifting procedure' for each component of the velocities. After the velocity $\hat{\mathbf{v}}$ and its derivatives $\hat{\mathbf{v}}_{x}$ and $\hat{\mathbf{v}}_{y}$ have been calculated, these values are taken to the non-advection phase.

\subsection{The non-advection phase}

The governing equations of the non-advection phase are

$$
\begin{aligned}
\frac{\mathbf{v}^{n+1}-\hat{\mathbf{v}}}{\Delta t}+\nabla p^{n+1} & =\frac{1}{R e} \nabla^{2} \mathbf{v}^{n} \\
\frac{\mathbf{v}_{x}^{n+1}-\hat{\mathbf{v}}_{x}}{\Delta t} & =-\left(\mathbf{v}_{x}^{n} \cdot \nabla\right) \mathbf{v}^{n}-\nabla p_{x}^{n+1}+\frac{1}{R e} \nabla^{2} \mathbf{v}_{x}^{n} \\
\frac{\mathbf{v}_{y}^{n+1}-\hat{\mathbf{v}}_{y}}{\Delta t} & =-\left(\mathbf{v}_{y}^{n} \cdot \nabla\right) \mathbf{v}^{n}-\nabla p_{y}^{n+1}+\frac{1}{R e} \nabla^{2} \mathbf{v}_{y}^{n}
\end{aligned}
$$

In order to avoid considering the pressure derivatives $p_{x}$ and $p_{y}$ as nodal variables and updating them for each time step in the second and third equations, these terms are replaced by the velocity 
terms from the first equation. Then, the second and third equations are written as

$$
\begin{aligned}
& \frac{\mathbf{v}_{x}^{n+1}-\hat{\mathbf{v}}_{x}}{\Delta t}=-\left(\mathbf{v}_{x}^{n} \cdot \nabla\right) \mathbf{v}^{n}+\frac{\partial}{\partial x}\left(\frac{\mathbf{v}^{n+1}-\hat{\mathbf{v}}}{\Delta t}\right) \\
& \frac{\mathbf{v}_{y}^{n+1}-\hat{\mathbf{v}}_{y}}{\Delta t}=-\left(\mathbf{v}_{y}^{n} \cdot \nabla\right) \mathbf{v}^{n}+\frac{\partial}{\partial y}\left(\frac{\mathbf{v}^{n+1}-\hat{\mathbf{v}}}{\Delta t}\right)
\end{aligned}
$$

The finite element solution is obtained by considering the weak form of these equations using the Galerkin method (the test functions correspond to the trial functions) over the volume $\Omega$ as

$$
\begin{aligned}
\int_{\Omega} \overline{\mathbf{v}}\left(\frac{\mathbf{v}^{n+1}-\hat{\mathbf{v}}}{\Delta t}\right) \mathrm{d} \Omega & =\int_{\Omega} \overline{\mathbf{v}}\left(\frac{1}{R e} \nabla^{2} \mathbf{v}^{n}-\nabla p^{n}\right) \mathrm{d} \Omega \\
\int_{\Omega} \overline{\mathbf{v}}\left(\frac{\mathbf{v}_{x}^{n+1}-\hat{\mathbf{v}}_{x}}{\Delta t}\right) \mathrm{d} \Omega & =\int_{\Omega} \overline{\mathbf{v}}\left[-\left(\mathbf{v}_{x}^{n} \cdot \nabla\right) \mathbf{v}^{n}+\frac{\partial}{\partial x}\left(\frac{\mathbf{v}^{n+1}-\hat{\mathbf{v}}}{\Delta t}\right)\right] \mathrm{d} \Omega \\
\int_{\Omega} \overline{\mathbf{v}}\left(\frac{\mathbf{v}_{y}^{n+1}-\hat{\mathbf{v}}_{y}}{\Delta t}\right) \mathrm{d} \Omega & =\int_{\Omega} \overline{\mathbf{v}}\left[-\left(\mathbf{v}_{y}^{n} \cdot \nabla\right) \mathbf{v}^{n}+\frac{\partial}{\partial y}\left(\frac{\mathbf{v}^{n+1}-\hat{\mathbf{v}}}{\Delta t}\right)\right] \mathrm{d} \Omega
\end{aligned}
$$

In the above equations, $\mathbf{v} \in V, p \in P, \overline{\mathbf{v}} \in V$, where $V$ and $P$ are appropriate Hilbert spaces, and the velocity vector $\mathbf{v}^{n+1}$ should satisfy the continuity equation $\nabla \cdot \mathbf{v}^{n+1}=0$. Note that the pressure term $\nabla p^{n+1}$ in (11) is taken to the right-hand side in (13) as $\nabla p^{n}$. In this way, the nodal pressures are not unknown nodal variables in (13). Instead, the pressure is updated from nodal velocities that are also iterated upon to satisfy the divergence-free condition at each time step, as explained below.

Equations (13) are solved using the Galerkin method with the 4-node element shown in Figure 1. We use the four corner points to interpolate the velocity and the velocity derivatives within each element, that is, we use

$$
\begin{gathered}
\mathbf{v}=\sum_{i=1}^{4} h_{i}^{v} \mathbf{v}_{i} \\
\mathbf{v}_{x}=\sum_{i=1}^{4} h_{i}^{v} \mathbf{v}_{x i} \\
\mathbf{v}_{y}=\sum_{i=1}^{4} h_{i}^{v} \mathbf{v}_{y i}
\end{gathered}
$$

where the trial functions in $V$ are defined in each element as

$$
\begin{aligned}
h_{1}^{v} & =\frac{1}{4}(1-\xi)(1-\eta) \\
h_{2}^{v} & =\frac{1}{4}(1+\xi)(1-\eta) \\
h_{3}^{v} & =\frac{1}{4}(1+\xi)(1+\eta) \\
h_{4}^{v} & =\frac{1}{4}(1-\xi)(1+\eta)
\end{aligned}
$$

with $-1 \leqslant \xi, \eta \leqslant 1$. 
First, the velocity $\mathbf{v}^{n+1}$ is obtained from the first equation in (13) and then the divergence of the velocity, $\nabla \cdot \mathbf{v}^{n+1}$, is calculated. This value is in general not zero and we need to iterate to update the velocities in order to have a zero divergence velocity field and to also calculate the updated pressure.

We use a standard simultaneous relaxation method to update the velocity vector and the pressure $[17,20]$. Let

$$
\begin{aligned}
\left(\mathbf{v}^{n+1}\right)^{(1)} & =\mathbf{v}^{n+1} \quad \text { of Equation (13) } \\
p^{(1)} & =p^{n}
\end{aligned}
$$

then we use for $m=1,2, \ldots$

$$
\begin{aligned}
\nabla^{2} \phi^{(m)} & =\nabla \cdot\left(\mathbf{v}^{n+1}\right)^{(m)} \\
p^{(m+1)} & =p^{(m)}+\phi^{(m)} / \Delta t \\
\left(\mathbf{v}^{n+1}\right)^{(m+1)} & =\left(\mathbf{v}^{n+1}\right)^{(m)}-\nabla \phi^{(m)}
\end{aligned}
$$

where the superscript $m$ represents the repeat level and $\phi$ is here the velocity potential.

We first calculate the divergence of the velocity, then we obtain the velocity potential $\phi$ from the first equation in (17). Using the second and third equations, the velocity and pressure are updated. We repeat the calculations until the velocity field satisfies the continuity equation; then we have

$$
\begin{aligned}
& \mathbf{v}^{n+1}=\left(\mathbf{v}^{n+1}\right)^{(m+1)} \\
& p^{n+1}=p^{(m+1)}
\end{aligned}
$$

This velocity $\mathbf{v}^{n+1}$ (in Equation (18)) is then used in the last two equations in (13) to obtain $\mathbf{v}_{x}^{n+1}$ and $\mathbf{v}_{y}^{n+1}$. The calculations for one time step are now complete.

For the next time step, we take the values $\mathbf{v}^{n+1}, p^{n+1}, \mathbf{v}_{x}^{n+1}$ and $\mathbf{v}_{y}^{n+1}$ to the advection phase and repeat the calculations.

However, as well-known, this element does not directly satisfy local mass and momentum conservations, and the satisfaction of the inf-sup condition for incompressible analysis is also of concern. For these reasons we next propose a 9-node element based on the interpolations used in the FCBI formulation [8].

\section{FORMULATION OF THE 9-NODE ELEMENT}

In this section, we present the formulation of a 9-node element for 2D incompressible fluid flows, that is based on the concepts used in the CIP and FCBI approaches.

\subsection{The governing equations}

The non-dimensional momentum and continuity equations and the spatial derivatives of the momentum equations are as given in Equations (1) and (2). 


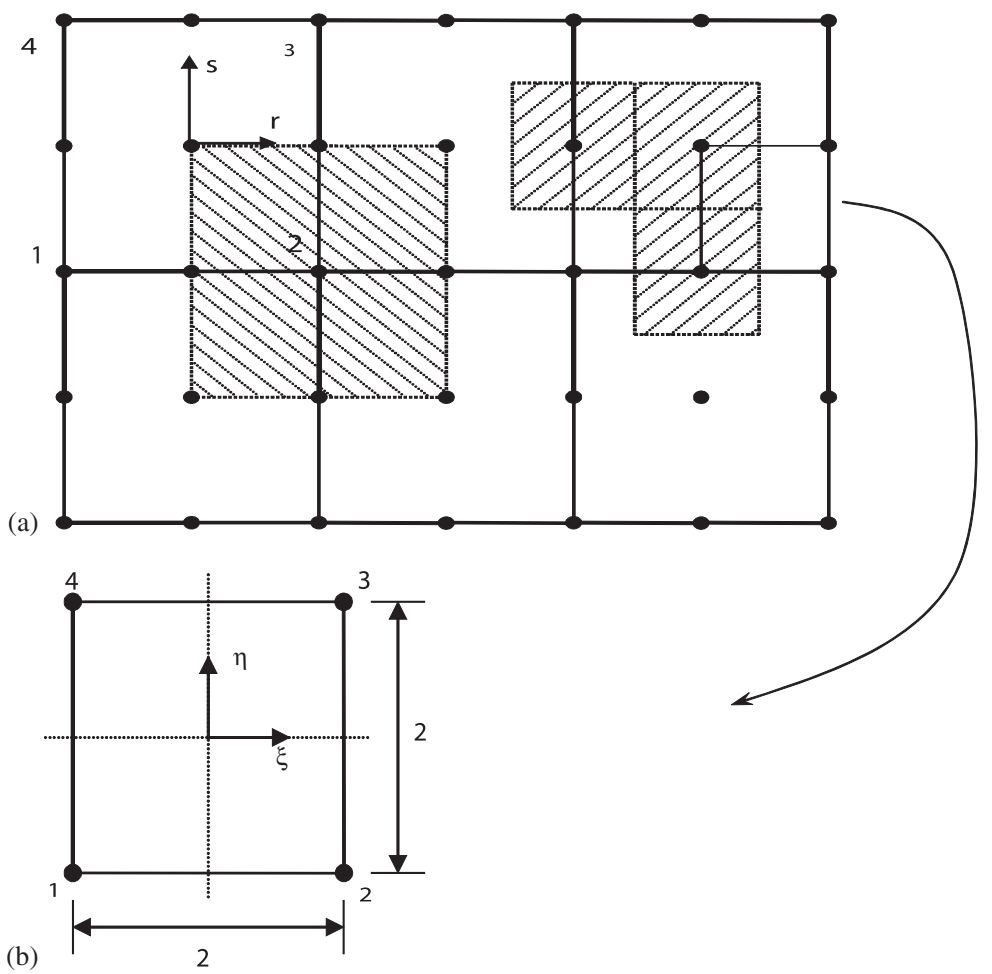

Figure 2. 9-node elements and the 4-node sub-elements.

Similar as in Section 2, we split the governing equations into an advection phase and a nonadvection phase. The advection phase is solved by the CIP method and the non-advection phase is solved as in Reference [8].

\subsection{The advection phase}

The governing equations of the advection phase are given in (3). These equations are solved as explained in Section 2 for a potential $\phi$ (see Equation (4)) using the third-order shape functions (7).

For the 9-node element, control volumes are employed in a Petrov-Galerkin formulation. Here, the control volumes shown in Figure 2 are implicitly employed by the use of step weight functions (see Equation (19)). Hence, for the advection terms, each 9-node element is divided into four 4-node sub-elements and the same interpolations as given in (8)-(10) are used within each sub-element.

\subsection{The non-advection phase}

As in Section 2, the governing equations are given in (11). Also, in order to avoid considering the pressure derivatives as nodal variables, these terms are replaced by the velocity terms as shown in (12). The finite element solution is obtained by considering a Petrov-Galerkin weak form of these equations and the continuity equation. 
Find $\mathbf{v} \in V$ and $p \in P$ such that

$$
\begin{aligned}
\int_{\Omega} w\left(\frac{\mathbf{v}^{n+1}-\hat{\mathbf{v}}}{\Delta t}+\nabla p^{n+1}\right) \mathrm{d} \Omega & =\int_{\Omega} w \frac{1}{R e} \nabla^{2} \mathbf{v}^{n} \mathrm{~d} \Omega \\
\int_{\Omega} q \nabla \cdot \mathbf{v}^{n+1} \mathrm{~d} \Omega & =0 \\
\int_{\Omega} w\left(\frac{\mathbf{v}_{x}^{n+1}-\hat{\mathbf{v}}_{x}}{\Delta t}\right) \mathrm{d} \Omega & =\int_{\Omega} w\left[-\left(\mathbf{v}_{x}^{n} \cdot \nabla\right) \mathbf{v}^{n}+\frac{\partial}{\partial x}\left(\frac{\mathbf{v}^{n+1}-\hat{\mathbf{v}}}{\Delta t}\right)\right] \mathrm{d} \Omega \\
\int_{\Omega} w\left(\frac{\mathbf{v}_{y}^{n+1}-\hat{\mathbf{v}}_{y}}{\Delta t}\right) \mathrm{d} \Omega & =\int_{\Omega} w\left[-\left(\mathbf{v}_{y}^{n} \cdot \nabla\right) \mathbf{v}^{n}+\frac{\partial}{\partial y}\left(\frac{\mathbf{v}^{n+1}-\hat{\mathbf{v}}}{\Delta t}\right)\right] \mathrm{d} \Omega
\end{aligned}
$$

where $w \in W$ and $q \in Q$, and $W$ and $Q$ are defined below, see also Reference [8].

Here, each 9-node element is divided into four 4-node sub-elements. For the 4-node sub-element shown in Figure 2, we use the four corner points to interpolate the velocity and hence the trial functions in $V$ are defined in each sub-element as

$$
\begin{aligned}
h_{1}^{v} & =\frac{1}{4}(1-\xi)(1-\eta) \\
h_{2}^{v} & =\frac{1}{4}(1+\xi)(1-\eta) \\
h_{3}^{v} & =\frac{1}{4}(1+\xi)(1+\eta) \\
h_{4}^{v} & =\frac{1}{4}(1-\xi)(1+\eta)
\end{aligned}
$$

with $-1 \leqslant \xi, \eta \leqslant 1$.

However, the trial functions in the space $P$ are given in each 9 -node element as, using the isoparametric co-ordinates $(r, s)$

$$
\begin{aligned}
h_{1}^{p} & =\frac{1}{4}(1-r)(1-s) \\
h_{2}^{p} & =\frac{1}{4}(1+r)(1-s) \\
h_{3}^{p} & =\frac{1}{4}(1+r)(1+s) \\
h_{4}^{p} & =\frac{1}{4}(1-r)(1+s)
\end{aligned}
$$

with $-1 \leqslant r, s \leqslant 1$. Hence, the velocity and pressure interpolations correspond to bilinear interpolations over the sub-elements and elements, respectively.

The elements in the space $Q$ are unit step functions. Referring to Figure 2(a), we have, for example, for the element at node 2

$$
h_{2}^{q}= \begin{cases}1 & \text { for }(r, s) \in[0,1] \times[-1,0] \\ 0 & \text { elsewhere }\end{cases}
$$


Similarly, the weight functions in the space $W$ are also unit step functions. Considering the sub-element shown in Figure 2(b), at node 1, for example,

$$
h_{1}^{w}= \begin{cases}1 & \text { for }(\xi, \eta) \in[-1,0] \times[-1,0] \\ 0 & \text { elsewhere }\end{cases}
$$

Then the velocity $\mathbf{v}$ and its derivatives and the pressure $p$ in (19) are expressed as

$$
\begin{aligned}
\mathbf{v} & =\sum_{i=1}^{4} h_{i}^{v} \mathbf{v}_{i} \\
\mathbf{v}_{x} & =\sum_{i=1}^{4} h_{i}^{v} \mathbf{v}_{x i} \\
\mathbf{v}_{y} & =\sum_{i=1}^{4} h_{i}^{v} \mathbf{v}_{y i} \\
p & =\sum_{j=1}^{4} h_{j}^{p} p_{j}
\end{aligned}
$$

\section{REMARKS ON THE SOLUTION OF THE GOVERNING EQUATIONS}

The given Equations (13) and (19) result in matrix equations for the finite element nodal unknowns. Note that first equations (3) are solved using the CIP solver (Equations (7)-(10)) to obtain $\hat{\mathbf{v}}$ and the spatial derivatives $\hat{\mathbf{v}}_{x}$ and $\hat{\mathbf{v}}_{y}$ for all nodal points. Then for the 4-node element, in the nonadvection phase, the nodal variables $\mathbf{v}^{n+1}$ and $p^{n+1}$ are calculated from the first equation in (13) and the continuity equation $\left(\nabla \cdot \mathbf{v}^{n+1}=0\right)$ using the relaxation method, as given in (17). Then we obtain the spatial derivatives $\mathbf{v}_{x}^{n+1}$ and $\mathbf{v}_{y}^{n+1}$ from the last two equations in (13). For the 9-node element, the $\mathbf{v}^{n+1}$ and $p^{n+1}$ are calculated from the first two equations in (19), and then we obtain the spatial derivatives $\mathbf{v}_{x}^{n+1}$ and $\mathbf{v}_{y}^{n+1}$ from the last two equations in (19). The calculations for one time step are complete now and for the next time step, we take the values $\mathbf{v}^{n+1}, p^{n+1}, \mathbf{v}_{x}^{n+1}$ and $\mathbf{v}_{y}^{n+1}$ to the advection phase and we repeat the calculations for the new time step.

We should also note that we used the simple Euler forward integration in time. For a steady-state solution the time integration needs to be marched until a steady-state is reached and the simple Euler forward method is adequate for this purpose. However, if an accurate transient solution is to be obtained, it is necessary to use a second-order accurate scheme in time, such as used in Reference [12], see also References [21,22].

\section{EXAMPLE SOLUTIONS}

To study the proposed element formulations, we consider the driven cavity flow problem shown in Figure 3. The boundary conditions are shown in this figure and the initial velocities are all zero except for the horizontal velocity $u=1$ at the upper side of the cavity. This problem is solved for two different cases; $R e=1000$ and 10000 . In both cases, the horizontal velocity at the vertical centerline, and the vertical velocity at the horizontal centreline are plotted and compared with the 


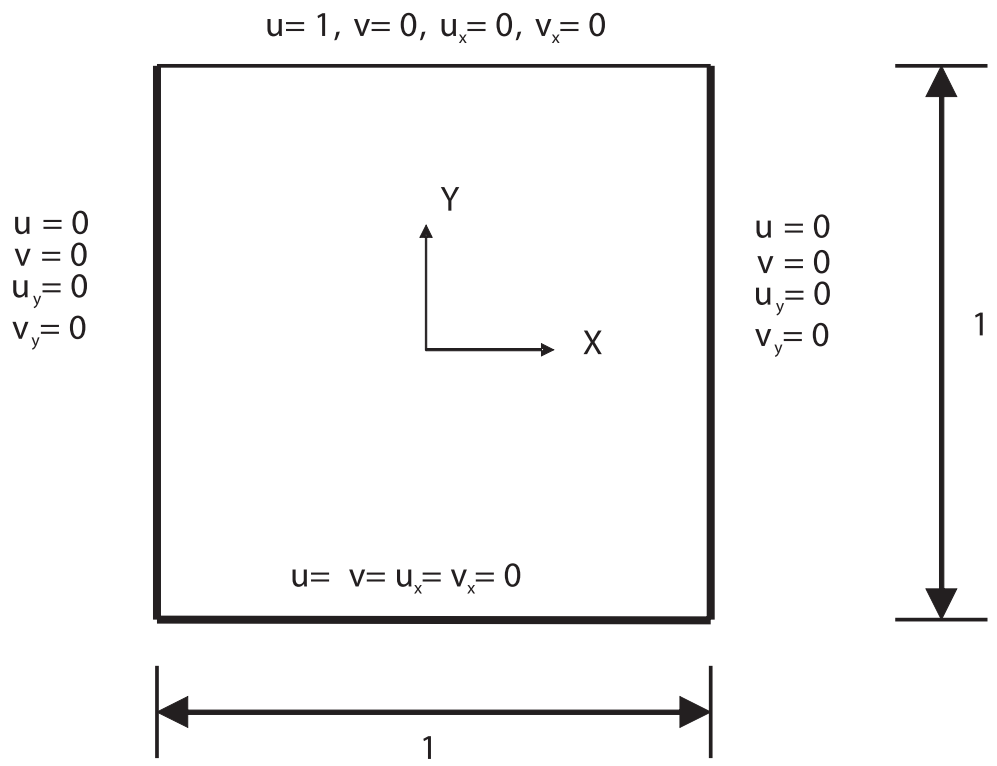

Figure 3 . The driven cavity flow problem.

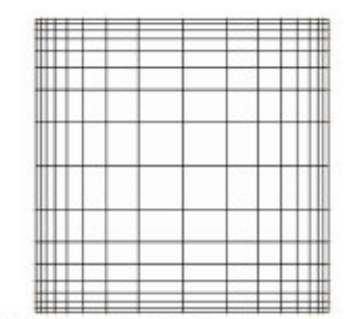

(a)

$16 \times 16$ elements

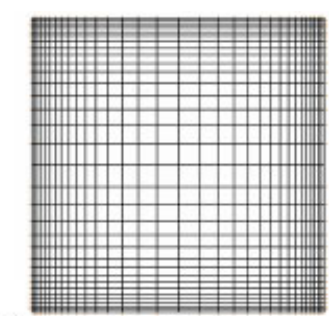

$32 \times 32$ elements

Figure 4. The non-uniform meshes of 9-node elements used for: (a) $R e=1000$; and (b) $R e=10000$.

solutions of Ghia et al. [23]. The meshes used are shown in Figures 4 and 5. Only steady-state solutions were pursued.

\subsection{The driven cavity flow problem for $R e=1000$}

For the solution of this problem, a non-uniform mesh of $16 \times 16$ 9-node elements (Figure 4(a)) has been used. For the time increment $\Delta t=0.005$, the flow is almost steady at $t=35$. The velocity profiles on the vertical and horizontal centre lines of the cavity are shown in Figure 6. In this figure, the solution obtained by Ghia et al. [23] is assumed to be very accurate.

The problem was also solved using the new 4-node element with a $30 \times 30$ mesh (Figure 5(a)). The velocities are slightly more accurate using, with this mesh, the new 4-node element. However, this solution involves also as an additional computational effort the iteration in Equations (17) and (18) in the non-advection phase. 


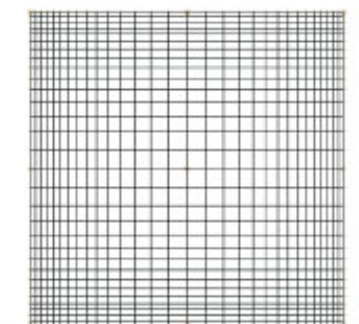

(a)

$30 \times 30$ elements

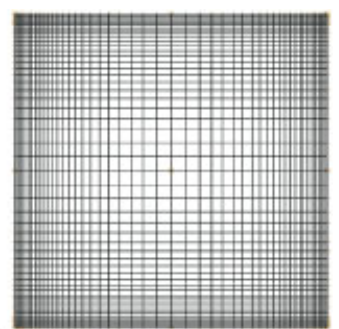

(b)

$50 \times 50$ elements

Figure 5. The non-uniform meshes of 4-node elements used for: (a) $R e=1000$; and (b) $R e=10000$.
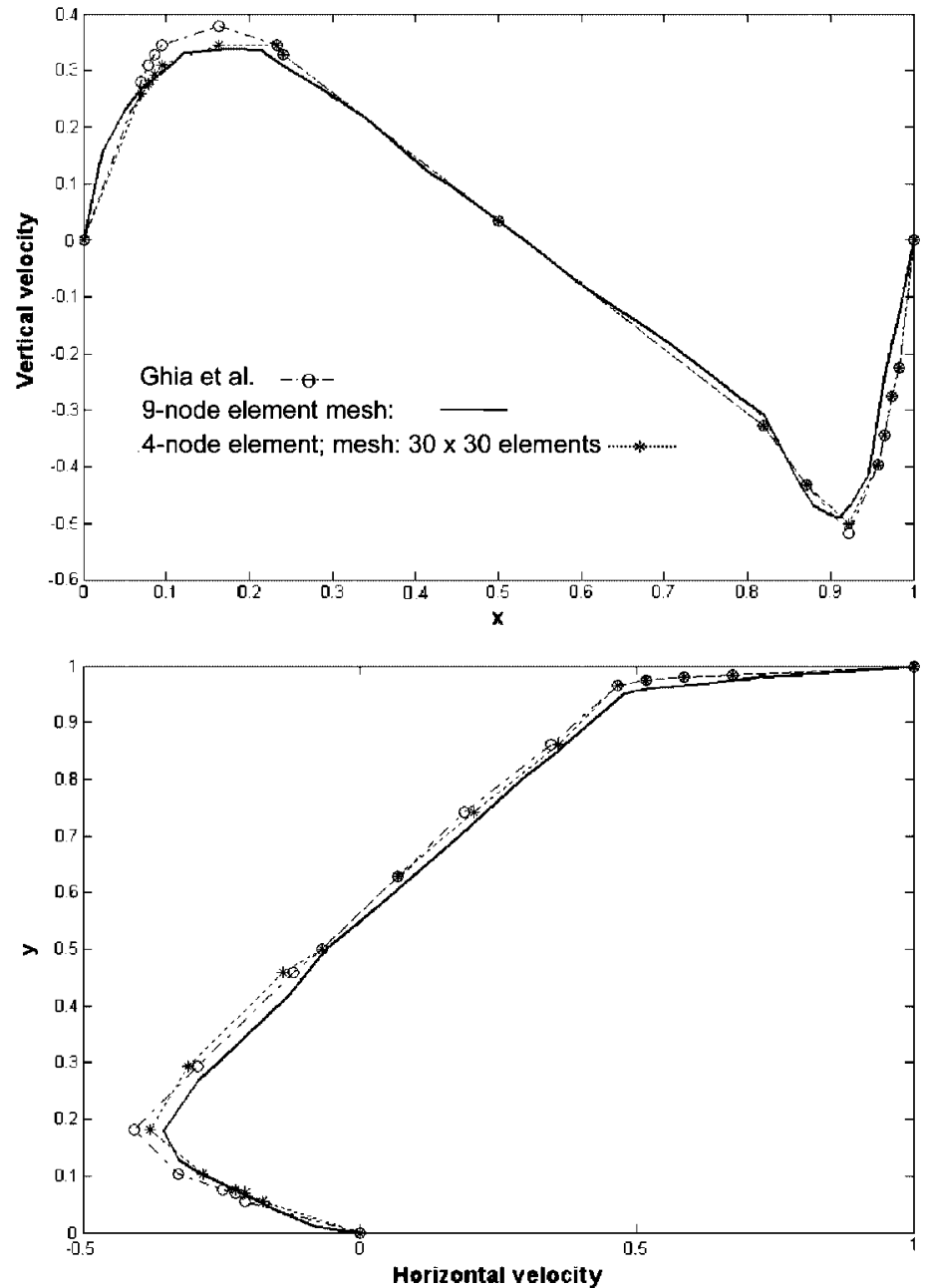

Figure 6. Velocity profiles for $R e=1000$. 

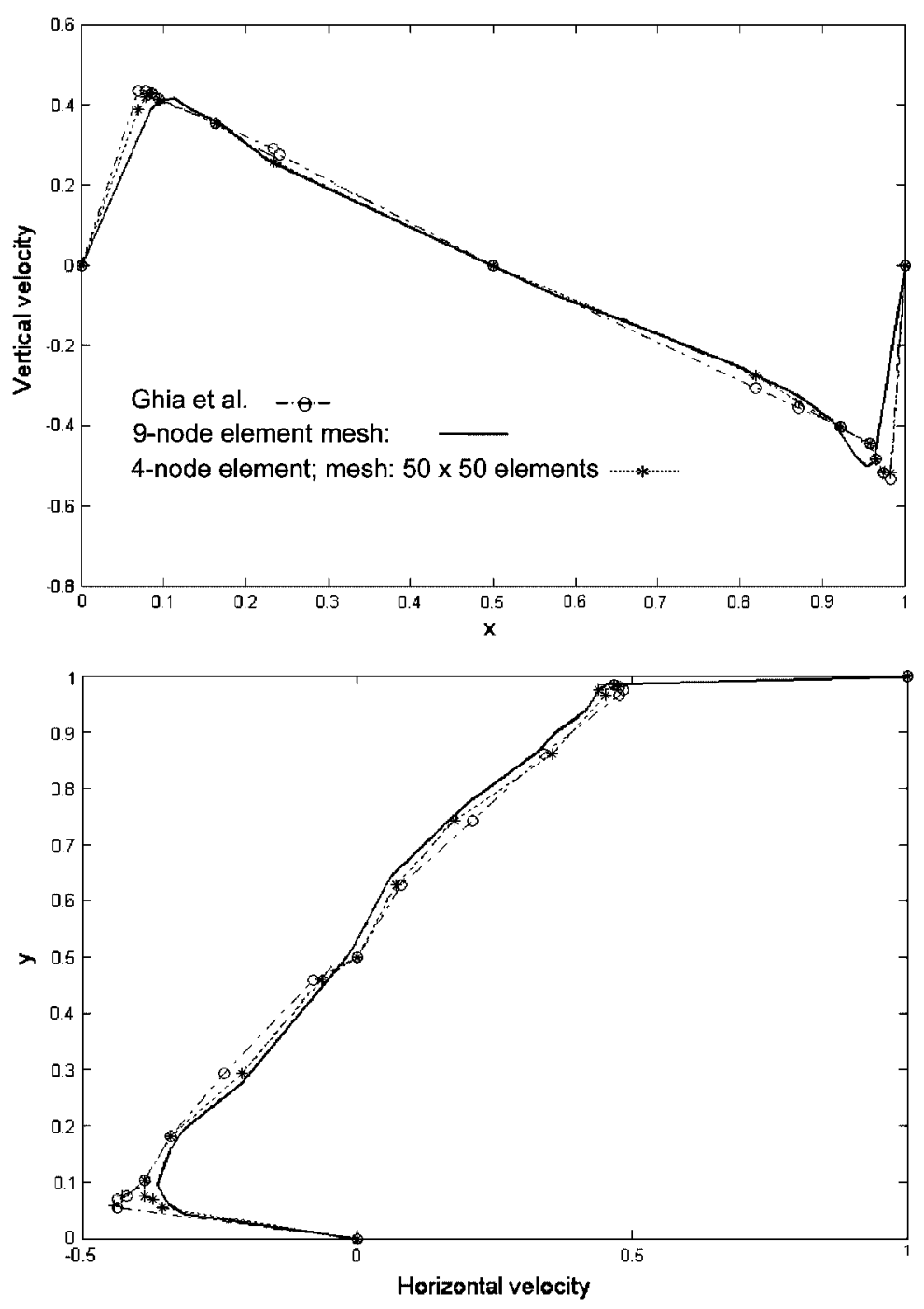

Figure 7. Velocity profiles for $R e=10000$.

It should be noted that although spatial derivatives of velocities are also considered as nodal variables using the new elements, the system of equations for velocities are solved separately from the system of equations for velocity derivatives (after the velocities have been obtained, the velocity derivatives are updated).

\subsection{The driven cavity flow problem for $R e=10000$}

For this solution the non-uniform mesh of $32 \times 32$ 9-node elements shown in Figure 4(b) with the time increment $\Delta t=0.002$ has been used. After the solution is steady, the velocity profiles on the 
vertical and horizontal centre axes of the cavity are as shown in Figure 7. In this figure, the Ghia et al. [23] solution is also given.

The problem was also solved using the new 4-node element with the $50 \times 50$ mesh shown in Figure 5(b). The velocities are slightly more accurate using, with this mesh, the new 4-node element.

\section{CONCLUDING REMARKS}

The objective in this paper was to present some research results obtained in our endeavours to develop stable and high accuracy finite elements for incompressible fluid flows. For this purpose we used in this paper the concepts of the CIP method and the FCBI approach to formulate two new elements.

The aims are to have elements that are stable in solutions even when coarse meshes are used in high Reynolds number flows (that is, when the element Reynolds numbers are very large) and achieve rapidly high accuracy as the mesh is refined. In this paper we only researched elements for $2 \mathrm{D}$ flows, but with the objective that a developed formulation can later also be applied to 3D conditions.

The 4-node and 9-node elements discussed in this paper showed in first tests quite good stability and accuracy, but are too expensive in applications. The element formulations clearly need to be further tested and also mathematically analysed. However, some concepts used are novel and valuable, and further research may well render the element formulations computationally more effective and thus become candidates for use in actual engineering practice.

\section{REFERENCES}

1. Bathe KJ (ed.). Computational fluid and solid mechanics. Proceedings of the Second MIT Conference on Computational Fluid and Solid Mechanics. Elsevier: Amsterdam, 2003.

2. Bathe KJ (ed.). Computational fluid and solid mechanics. Proceedings of the Third MIT Conference on Computational Fluid and Solid Mechanics. Elsevier: Amsterdam, 2005.

3. Gresho P, Sani RL. Incompressible Flow and the Finite Element Method. Wiley: New York, 2000.

4. Iannelli J. Characteristics Finite Element Methods in Computational Fluid Dynamics. Springer: Berlin, 2006.

5. Grätsch T, Bathe KJ. A posteriori error estimation techniques in practical finite element analysis. Computers and Structures 2005; 83:235-265.

6. Grätsch T, Bathe KJ. Goal-oriented error estimation in the analysis of fluid flows with structural interactions. Computer Methods in Applied Mechanics and Engineering 2006; 195:5673-5684.

7. Bathe KJ, Pontaza JP. A flow-condition-based interpolation mixed finite element procedure for higher Reynolds number fluid flows. Mathematical Models and Methods in Applied Sciences 2002; 12(4):525-539.

8. Bathe KJ, Zhang H. A flow-condition-based interpolation finite element procedure for incompressible fluid flows. Computers and Structures 2002; 80:1267-1277.

9. Kohno H, Bathe KJ. Insight into the flow-condition-based interpolation finite element approach: solution of steady-state advection-diffusion problems. International Journal for Numerical Methods in Engineering 2005; 63:197-217.

10. Kohno H, Bathe KJ. A flow-condition-based interpolation finite element procedure for triangular grids. International Journal for Numerical Methods in Fluids 2005; 49:849-875.

11. Kohno H, Bathe KJ. A nine-node quadrilateral FCBI element for incompressible fluid flows. Communications in Numerical Methods in Engineering 2006; 22:917-931.

12. Bathe KJ, Zhang H. Finite element developments for general fluid flows with structural interactions. International Journal for Numerical Methods in Engineering 2004; 60:213-232. 
13. Yabe T, Aoki T. A universal solver for hyperbolic equations by cubic-polynomial interpolation I. One-dimensional solver. Computer Physics Communications 1991; 66:219-232.

14. Yabe T, Aoki T. A universal solver for hyperbolic equations by cubic-polynomial interpolation II. Two- and three-dimensional solver. Computer Physics Communications 1991; 66:233-242.

15. Yabe T, Xiao F. Frontier of multi-phase flow analysis and fluid-structure interaction. Computers and Structures 2005; 83(6-7):395.

16. Takizawa K, Yabe T, Chino M, Kawai T, Wataji K, Hoshino H, Watanabe T. Simulation and experiment on swimming fish and skimmer by CIP method. Computers and Structures 2005; 83(6-7):397-408.

17. Makihara T, Shibata E, Tanahashi T. Application of the GSMAC-CIP method to the incompressible Navier-Stokes equation at high Reynolds numbers. International Journal of Computational Fluid Dynamics 1999; 12:301-314.

18. Ikeda H, Shioda K. Numerical analysis of natural convection at high Rayleigh numbers using the GSMAC-CIP method. Ninth Computational Mechanics Conference 1996; 279 (in Japanese).

19. Makihara T, Tanahashi T, Matsumoto M. Finite element method for CIP method (verification of three-D advection equation). Transactions of the Japan Society for Mechanical Engineering 1997; B63(609):1612 (in Japanese).

20. Makihara T, Kakuta Y, Tanahashi T. The GSMAC-CIP method. Computational Fluid Dynamics Journal 1999; 8(1):19-25.

21. Bathe KJ, Baig MMI. On a composite implicit time integration procedure for nonlinear dynamics. Computers and Structures 2005; 83:2513-2524.

22. Bathe KJ. Conserving energy and momentum in nonlinear dynamics: a simple implicit time integration scheme. Computers and Structures, in press.

23. Ghia U, Ghia KN, Shin CT. High-Re solutions for incompressible flow using the Navier-Stokes equations and a multigrid method. Journal of Computational Physics 1982; 48:387-411. 